\title{
On multicolor Ramsey number of paths versus cycles
}

\author{
Gholam Reza Omidi ${ }^{1}$ \\ Department of Mathematical Sciences \\ Isfahan University of Technology \\ Isfahan, 84156-83111, Iran \\ and \\ School of Mathematics \\ Institute for Research in Fundamental Sciences \\ Tehran, 19395-5746, Iran \\ romidi@cc.iut.ac.ir
}

Ghaffar Raeisi

Department of Mathematical Sciences

Isfahan University of Technology

Isfahan, 84156-83111, Iran

g.raeisi@math.iut.ac.ir

Submitted: Sep 5, 2010; Accepted: Jan 10, 2011; Published: Jan 26, 2011

Mathematics Subject Classifications: 05C15, 05 C55.

\begin{abstract}
Let $G_{1}, G_{2}, \ldots, G_{t}$ be graphs. The multicolor Ramsey number $R\left(G_{1}, G_{2}, \ldots, G_{t}\right)$ is the smallest positive integer $n$ such that if the edges of a complete graph $K_{n}$ are partitioned into $t$ disjoint color classes giving $t$ graphs $H_{1}, H_{2}, \ldots, H_{t}$, then at least one $H_{i}$ has a subgraph isomorphic to $G_{i}$. In this paper, we provide the exact value of $R\left(P_{n_{1}}, P_{n_{2}}, \ldots, P_{n_{t}}, C_{k}\right)$ for certain values of $n_{i}$ and $k$. In addition, the exact values of $R\left(P_{5}, C_{4}, P_{k}\right), R\left(P_{4}, C_{4}, P_{k}\right), R\left(P_{5}, P_{5}, P_{k}\right)$ and $R\left(P_{5}, P_{6}, P_{k}\right)$ are given. Finally, we give a lower bound for $R\left(P_{2 n_{1}}, P_{2 n_{2}}, \ldots, P_{2 n_{t}}\right)$ and we conjecture that this lower bound is the exact value of this number. Moreover, some evidence is given for this conjecture.
\end{abstract}

\section{Introduction}

In this paper, we are only concerned with undirected simple finite graphs and we follow [1] for terminology and notations not defined here. The complement graph of a graph $G$ is denoted by $\bar{G}$. As usual, the complete graph of order $p$ is denoted by $K_{p}$ and a complete bipartite graph with partite set $(X, Y)$ such that $|X|=m$ and $|Y|=n$ is denoted by $K_{m, n}$. Throughout this paper, we denote a cycle and a path on $m$ vertices by $C_{m}$ and $P_{m}$, respectively. Also for a 3-edge coloring (say green, blue and red) of a graph $G$, we denote by $G^{g}$ (resp. $G^{b}$ and $G^{r}$ ) the subgraph induced by the edges of color green (resp. blue and red).

\footnotetext{
${ }^{1}$ This research was in part supported by a grant from IPM (No. 89050037)
} 
Let $G_{1}, G_{2}, \ldots, G_{t}$ be graphs. The multicolor Ramsey number $R\left(G_{1}, G_{2}, \ldots, G_{t}\right)$, is the smallest positive integer $n$ such that if the edges of a complete graph $K_{n}$ are partitioned into $t$ disjoint color classes giving $t$ graphs $H_{1}, H_{2}, \ldots, H_{t}$, then at least one $H_{i}$ has a subgraph isomorphic to $G_{i}$. The existence of such a positive integer is guaranteed by Ramsey's classical result [12]. Since their time, particulary since the 1970's, Ramsey theory has grown into one of the most active areas of research within combinatorics, overlapping variously with graph theory, number theory, geometry and logic.

For $t \geq 3$, there is a few results about multicolor Ramsey number $R\left(G_{1}, G_{2}, \ldots, G_{t}\right)$. A survey including some results on Ramsey number of graphs, can be found in [11]. The multicolor Ramsey numbers $R\left(P_{n_{1}}, P_{n_{2}}, \ldots, P_{n_{t}}\right)$ and $R\left(P_{n_{1}}, P_{n_{2}}, \ldots, C_{n_{t}}\right)$ are not known for $t \geq 3$. In the case $t=2$, a well-known theorem of Gerencsér and Gyárfás [9] states that $R\left(P_{n}, P_{m}\right)=n+\left\lfloor\frac{m}{2}\right\rfloor-1$, where $n \geq m \geq 2$. Faudree and Schelp in [7] determined $R\left(P_{n_{1}}, P_{2 n_{2}+\delta}, \ldots, P_{2 n_{t}}\right)$ where $\delta \in\{0,1\}$ and $n_{1}$ is sufficiently large. In addition, they determined $R\left(P_{n_{1}}, P_{n_{2}}, P_{n_{3}}\right)$ for the case $n_{1} \geq 6\left(n_{2}+n_{3}\right)^{2}$ and they conjectured that

$$
R\left(P_{n}, P_{n}, P_{n}\right)= \begin{cases}2 n-1 & \text { if } n \text { is odd } \\ 2 n-2 & \text { if } n \text { is even. }\end{cases}
$$

This conjecture was established by Gyárfás et al. [10] for sufficiently large $n$. In asymptotic form, this was proved by Figaj and Luczak in [8] as a corollary of more general results about the asymptotic results of the Ramsey number for three long even cycles.

Recently, determination of some exact values of Ramsey numbers of type $R\left(P_{i}, P_{j}, C_{k}\right)$ such as $R\left(P_{4}, P_{4}, C_{k}\right), R\left(P_{4}, P_{6}, C_{k}\right)$ and $R\left(P_{3}, P_{5}, C_{k}\right)$ have been investigated. For more details related to three-color Ramsey numbers for paths versus a cycle, see [3, 4, 5, 13]. In this paper, we provide the exact value of the Ramsey numbers $R\left(P_{n_{1}}, P_{n_{2}}, \ldots, P_{n_{t}}, C_{k}\right)$ for certain values of $n_{i}$ and $k$ and then we determine the exact values of some three-color Ramsey numbers of type $R\left(P_{i}, P_{j}, C_{k}\right)$ as corollaries of our result. Moreover, we determine the exact value of the multicolor Ramsey number $R\left(P_{n_{1}}, P_{n_{2}}, \ldots, P_{n_{t}}, C_{k}\right)$, if at most one $n_{i}$ is odd and $k$ is sufficiently large. Consequently, we obtain an improvement of the result of Faudree and Schelp [7] on multicolor Ramsey number $R\left(P_{n_{1}}, P_{2 n_{2}+\delta}, \ldots, P_{2 n_{t}}\right)$. In addition, we determine the exact values of some three-color Ramsey numbers such as $R\left(P_{5}, C_{4}, P_{k}\right), R\left(P_{4}, C_{4}, P_{k}\right), R\left(P_{5}, P_{5}, P_{k}\right)$ and $R\left(P_{5}, P_{6}, P_{k}\right)$. Finally, we give a lower bound for $R\left(P_{2 n_{1}}, P_{2 n_{2}}, \ldots, P_{2 n_{t}}\right)$ and we conjecture that, with giving some evidences, this lower bound is the exact value of this number.

\section{Multicolor Ramsey number $R\left(P_{n_{1}}, P_{n_{2}}, \ldots, P_{n_{t}}, C_{k}\right)$}

In this section, we determine the exact value of $R\left(P_{n_{1}}, P_{n_{2}}, \ldots, P_{n_{t}}, C_{k}\right)$ when at most one of $n_{i}$ is odd and $k$ is sufficiently large. Also, the exact values of some known three-color Ramsey numbers of type $R\left(P_{i}, P_{j}, C_{k}\right)$ are given as some corollaries. For this purpose, we 
need some definitions and notations. A graph $G$ is called $H$-free if it does not contain $H$ as a subgraph. The notation $e x(p, H)$ is defined the maximum number of edges in a $H$-free graph on $p$ vertices. It is well known that [6] $e x\left(p, P_{n}\right) \leq \frac{(n-2)}{2} p$, for every $n$. Moreover, $\operatorname{ex}\left(p, C_{k}\right)$ is known for some values of $p$ and $k$. The following theorem can be found in the appendix IV of [1].

Theorem 2.1 ([1]) Assume that $k \geq \frac{1}{2}(p+3)$. Then

$$
\operatorname{ex}\left(p, C_{k}\right)=\left(\begin{array}{c}
p-k+2 \\
2
\end{array}\right)+\left(\begin{array}{c}
k-1 \\
2
\end{array}\right)
$$

Now, we are ready to establish the main result of this section.

Theorem 2.2 Let $k \geq n_{1} \geq n_{2} \geq \cdots \geq n_{t} \geq 3$ and $l \geq 1$ be a positive integer that can be written as $l=\sum_{i=1}^{t} x_{i}$ for some $x_{i}$ such that $2 x_{i}+1<n_{i}$. Then in the following cases, we have $R\left(P_{n_{1}}, P_{n_{2}}, \ldots, P_{n_{t}}, C_{k}\right)=k+l$.

(i) If $k \geq 2 l^{2}+5 l+5$ and $\sum_{i=1}^{t} n_{i}=2 l+2 t+1$,

(ii) If $k \geq l^{2}+2 l+3$ and $\sum_{i=1}^{t} n_{i}=2 l+2 t$.

Proof. Let $R$ denote the multicolor Ramsey number $R\left(P_{n_{1}}, P_{n_{2}}, \ldots, P_{n_{t}}, C_{k}\right)$. By Theorem 2.1, we obtain that ex $\left(k+l, C_{k}\right)=\frac{1}{2}\left(k^{2}+l^{2}-3 k+3 l+4\right)$ where $k \geq l+3$. Clearly $R \leq k+l$ if the following inequality holds.

$$
\sum_{i=1}^{t} e x\left(k+l, P_{n_{i}}\right)+e x\left(k+l, C_{k}\right)<\left(\begin{array}{c}
k+l \\
2
\end{array}\right) .
$$

In the other words, $R \leq k+l$ if

$$
\frac{k+l}{2}\left(\sum_{i=1}^{t} n_{i}-2 t\right)+\frac{1}{2}\left(k^{2}+l^{2}-3 k+3 l+4\right)<\left(\begin{array}{c}
k+l \\
2
\end{array}\right),
$$

or simply

$$
\sum_{i=1}^{t} n_{i}<(2 t+2 l+2)-\frac{2 l^{2}+6 l+4}{k+l}
$$

In each case of the theorem, inequality (1) holds and so $R \leq k+l$. Now consider the graph $K_{k-1} \cup K_{l}$ and partition the vertices of $K_{l}$ into $t$ classes $V_{1}, V_{2}, \ldots, V_{t}$ such that $\left|V_{i}\right|=x_{i}$, $1 \leq i \leq t$. Color the edges of $K_{k-1}$ and $K_{l}$ by color $\alpha_{t+1}$ and also color the edges having an end vertex in $V_{i}, 1 \leq i \leq t$, and one in $K_{k-1}$ by color $\alpha_{i}$. Since for $i=1,2, \ldots, t$, the inequality $2\left|V_{i}\right|+1<n_{i}$ holds, this coloring of $K_{k+l-1}$ contains no $P_{n_{i}}$ in color $\alpha_{i}$, $1 \leq i \leq t$, and no $C_{k}$ in color $\alpha_{t+1}$. This means that $R \geq k+l$, which completes the proof.

In the following theorem, we determine the exact value of $R\left(P_{2 n_{1}}, P_{2 n_{2}}, \ldots, P_{2 n_{t}}, C_{k}\right)$ for sufficiently large $k$. 
Theorem 2.3 Assume that $\delta \in\{0,1\}$ and $\Sigma$ denotes $\sum_{i=1}^{t}\left(n_{i}-1\right)$. Then

$$
R\left(P_{2 n_{1}+\delta}, P_{2 n_{2}}, \ldots, P_{2 n_{t}}, C_{k}\right)=k+\Sigma,
$$

where $k \geq \Sigma^{2}+2 \Sigma+3$ if $\delta=0$ and $k \geq 2 \Sigma^{2}+5 \Sigma+5$, otherwise.

Proof. The assertion holds from Theorem 2.2 where $x_{i}=n_{i}-1$ for $1 \leq i \leq t$.

As an application of Theorem 2.3, we have the following corollary which determine some known three-color Ramsey numbers of small paths versus a cycle.

Corollary 2.4 Let $k$ be a positive integer. Then

(i) ([3]) $R\left(P_{4}, P_{4}, C_{k}\right)=k+2$ for $k \geq 11$,

(ii) ([4]) $R\left(P_{3}, P_{4}, C_{k}\right)=k+1$ for $k \geq 12$,

(iii) ([13]) $R\left(P_{4}, P_{5}, C_{k}\right)=k+2$ for $k \geq 23$,

(iv) ([13]) $R\left(P_{4}, P_{6}, C_{k}\right)=k+3$ for $k \geq 18$.

We end this section by giving the following consequent of Theorem 2.3.

Corollary 2.5 Let $k$ be a positive integer. Then

(i) $R\left(P_{3}, P_{6}, C_{k}\right)=k+2$ for $k \geq 23$,

(ii) $R\left(P_{6}, P_{6}, C_{k}\right)=R\left(P_{4}, P_{8}, C_{k}\right)=k+4$ for $k \geq 27$,

(iii) $R\left(P_{6}, P_{7}, C_{k}\right)=k+4$ for $k \geq 57$.

\section{Some three-color Ramsey numbers}

In this section, we provide the exact values of some three-color Ramsey numbers such as $R\left(P_{5}, C_{4}, P_{m}\right), R\left(P_{4}, C_{4}, P_{m}\right), R\left(P_{5}, P_{5}, P_{m}\right)$ and $R\left(P_{5}, P_{6}, P_{m}\right)$. First, we recall a result of Faudree and Schelp.

Theorem 3.1 ([7]) If $G$ is a graph with $|V(G)|=n t+r$ where $0 \leq r<n$ and $G$ contains no path on $n+1$ vertices, then $|E(G)| \leq t\left(\begin{array}{l}n \\ 2\end{array}\right)+\left(\begin{array}{l}r \\ 2\end{array}\right)$ with equality if and only if either $G \cong t K_{n} \cup K_{r}$ or if $n$ is odd, $t>0$ and $r=(n \pm 1) / 2$

$$
G \cong l K_{n} \cup\left(K_{(n-1) / 2}+\bar{K}_{((n+1) / 2+(t-l-1) n+r)}\right),
$$

for some $0 \leq l<t$.

By Theorem 3.1, it is easy to obtain the following corollary. 
Corollary 3.2 For all integer $n \geq 3$,

$$
\begin{aligned}
& e x\left(n, P_{4}\right)= \begin{cases}n & \text { if } n=0(\bmod 3), \\
n-1 & \text { if } n=1,2(\bmod 3) .\end{cases} \\
& e x\left(n, P_{5}\right)= \begin{cases}3 n / 2 & \text { if } n=0(\bmod 4), \\
3 n / 2-2 & \text { if } n=2(\bmod 4), \\
(3 n-3) / 2 & \text { if } n=1,3 \bmod 4 .\end{cases} \\
& e x\left(n, P_{6}\right)= \begin{cases}2 n & \text { if } n=0(\bmod 5), \\
2 n-2 & \text { if } n=1,4(\bmod 5), \\
2 n-3 & \text { if } n=2,3 \bmod 5 .\end{cases}
\end{aligned}
$$

In order to prove the main results of this section, we need some lemmas.

Lemma 3.3 ([13]) Let $G$ be a complete bipartite graph $K_{3,4}$ with two partite sets $X$ and $Y$ where $|X|=3$ and $|Y|=4$. If each edge of $G$ is colored green or blue, then $G$ contains either a green $P_{5}$ or a blue $C_{4}$.

Lemma 3.4 ([13]) Let $G$ be a graph obtained by removing two edges from $K_{6}$. If each edge of $G$ is colored green or blue, then $G$ contains either a green $P_{5}$ or a blue $C_{4}$.

Using Lemma 3.3, we have the following lemma.

Lemma 3.5 Let $G$ be a complete bipartite graph $K_{3,5}$ with two partite sets $X$ and $Y$ where $|X|=3$ and $|Y|=5$. If each edge of $G$ is colored green or blue, then $G$ contains a monochromatic graph $P_{5}$.

Proof. Let $X=\left\{x_{1}, x_{2}, x_{3}\right\}$ and $Y=\left\{y_{1}, y_{2}, y_{3}, y_{4}, y_{5}\right\}$. By Lemma 3.3, G must contain a green $P_{5}$ or a blue $C_{4}$. If a green $P_{5}$ occur, we are done. So let $G$ contains a blue $C_{4}$ on vertices $x_{1}, y_{1}, x_{2}, y_{2}$, in this order. If one of the edges $x_{i} y_{j}, i \in\{1,2\}$ and $j \in\{3,4,5\}$, is blue we obtain a blue $P_{5}$. Otherwise, we may assume that these edges are all in green color. Clearly this gives a green $P_{5}=y_{5} x_{2} y_{4} x_{1} y_{3}$, which completes the proof.

Now, we use previous results to prove the following lemma, which help us to calculate the three-color Ramsey number $R\left(P_{5}, C_{4}, P_{m}\right)$.

Lemma 3.6 Let $m \geq 5$ and the edges of $K_{m+2}$ be colored with colors green, blue and red such that $G^{r}$ contains a copy of $P_{m-1}$ as a subgraph. Then $K_{m+2}$ contains either a green $P_{5}$, a blue $C_{4}$ or a red $P_{m}$. 

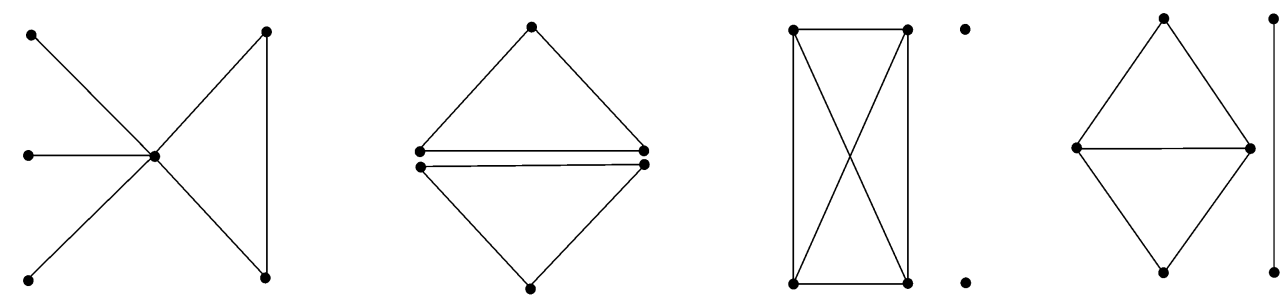

Fig. 1: $P_{5}$-free graphs on 6 vertices and 6 edges

Proof. Assume that $V\left(K_{m+2}\right)=\left\{v_{1}, v_{2}, \ldots, v_{m+2}\right\}$ and $P=v_{1} v_{2} \ldots v_{m-1}$ is the desired copy of $P_{m-1}$ in $G^{r}$. We suppose that $G^{r}$ contains no copy of $P_{m}$, then we prove that $K_{m+2}$ contains either a green $P_{5}$ or a blue $C_{4}$. First assume that $v_{1} v_{m-1} \in E\left(G^{r}\right)$. If one of the vertices $v_{m}, v_{m+1}$ or $v_{m+2}$ is adjacent to $P$ in $G^{r}$ then we obtain a red $P_{m}$, a contradiction. So each edge between $\left\{v_{m}, v_{m+1}, v_{m+2}\right\}$ and $P$ is colored green or blue. Since $m \geq 5$, we obtain the complete bipartite graph $K_{3,4}$ on two partite set $X=\left\{v_{m}, v_{m+1}, v_{m+2}\right\}$ and $Y=\left\{v_{1}, v_{2}, v_{m-2}, v_{m-1}\right\}$ with all edges are colored green or blue. Using Lemma 3.3, we obtain a green $P_{5}$ or a blue $C_{4}$. Hence we may assume that $v_{1} v_{m-1} \notin E\left(G^{r}\right)$. Also all edges between $\left\{v_{1}, v_{m-1}\right\}$ and $\left\{v_{m}, v_{m+1}, v_{m+2}\right\}$ are colored by green or blue, otherwise we have a red $P_{m}$. Let $H$ be a subgraph of $G^{r}$ induced by the edges of color red on vertices $\left\{v_{m}, v_{m+1}, v_{m+2}\right\}$. We have the following cases.

Case 1. $|E(H)|=0$.

Since $|E(H)|=0$, all edges between vertices $T=\left\{v_{1}, v_{m-1}, v_{m}, v_{m+1}, v_{m+2}\right\}$ are colored by green or blue. We find a vertex $v \in P$ such that $T \cup\{v\}$ are the vertices of a complete graph on six vertices with at most two red edges and then we use Lemma 3.4, which guaranties the existence of a green $P_{5}$ or a blue $C_{4}$. If there is a vertex $v \in P-\left\{v_{1}, v_{m-1}\right\}$ such that for each $i \in\{m, m+1, m+2\}, v v_{i} \notin E\left(G^{r}\right)$, then this vertex is the desired vertex. Also note that two consecutive vertices of $P$ are not adjacent in $G^{r}$ to a vertex in $\left\{v_{m}, v_{m+1}, v_{m+2}\right\}$, otherwise we have a red copy of $P_{m}$, a contradiction. So, without loss of generality, let $v_{2} v_{m}, v_{3} v_{m+1} \in E\left(G^{r}\right)$. If $v_{3} v_{1} \in E\left(G^{r}\right)$, then $P_{m}=v_{m} v_{2} v_{1} v_{3} v_{4} \ldots v_{m-1}$ is a red $P_{m}$ and so $v_{3} v_{1} \notin E\left(G^{r}\right)$. By the same argument, $v_{2} v_{m-1} \notin G^{r}$. Now let $v=v_{3}$ if $v_{3} v_{m+2} \notin E\left(G^{r}\right)$ and $v=v_{2}$ otherwise. In any case, $T \cup\{v\}$ form a complete graph on six vertices with at most two red edges.

Case 2. $|E(H)|=1$.

Let $E(H)=\left\{v_{m} v_{m+1}\right\}$. Since $P_{m} \nsubseteq G^{r}, v_{2}$ (also $v_{m-2}$ ) is not adjacent to $v_{m}$ or $v_{m+1}$ in $G^{r}$. If $v_{2} v_{m-1}, v_{1} v_{3} \in E\left(G^{r}\right)$, then $G^{r}$ contains $C_{m-1}=v_{2} v_{1} v_{3} \ldots v_{m-1} v_{2}$ and so each edge between $X=\left\{v_{m}, v_{m+1}, v_{m+2}\right\}$ and $Y=\left\{v_{1}, v_{2}, v_{m-2}, v_{m-1}\right\}$ is colored green or blue, since $P_{m} \nsubseteq G^{r}$. Using Lemma 3.3, we obtain either a green $P_{5}$ or a blue $C_{4}$. Therefore if $v_{2} v_{m-1} \in E\left(G^{r}\right)$, then $v_{1} v_{3} \notin E\left(G^{r}\right)$. Now, assume that $v_{2} v_{m+2} \notin E\left(G^{r}\right)$. If $v_{2} v_{m-1} \notin E\left(G^{r}\right)$, then $\left\{v_{1}, v_{2}, v_{m-1}, v_{m}, v_{m+1}, v_{m+2}\right\}$ are the vertices of a complete 
graph on six vertices with at most two red edges. Also if $v_{2} v_{m-1} \in E\left(G^{r}\right)$, then for each $i \in\{m, m+1, m+2\}, v_{3} v_{i} \notin E\left(G^{r}\right)$, otherwise we have a red $P_{m}$. In this case $\left\{v_{1}, v_{3}, v_{m-1}, v_{m}, v_{m+1}, v_{m+2}\right\}$ are the vertices of a complete graph on six vertices with at most two red edges. Using Lemma 3.4, we obtain a green $P_{5}$ or blue $C_{4}$, as desired. So we may assume that $v_{2} v_{m+2}$ is an edge of $G^{r}$. If $m=5$, then $\left\{v_{1}, v_{3}, v_{m-1}, v_{m}, v_{m+1}, v_{m+2}\right\}$ are the vertices of a complete graph on six vertices such that each edge is colored green or blue except at most two edges. Now let $m \geq 6$. By the same argument, we may assume that $v_{m-2} v_{m+2} \in E\left(G^{r}\right)$. If for some $i \in\{m, m+1, m+2\}, v_{3} v_{i} \in E\left(G^{r}\right)$, then we obtain $P_{m}=v_{1} v_{2} v_{m+2} v_{m-2} \ldots v_{3} v_{i}$ in $G^{r}$. Also if $v_{1} v_{3} \in E\left(G^{r}\right)$, then we obtain a copy of $P_{m}=v_{m+2} v_{2} v_{1} v_{3} \ldots v_{m-1}$ in $G^{r}$, a contradiction. Hence $\left\{v_{1}, v_{3}, v_{m-1}, v_{m}, v_{m+1}, v_{m+2}\right\}$ are the vertices of a complete graph on six vertices such that each edge is colored green or blue except at most two edges. Lemma 3.4, guaranties the existence of a green $P_{5}$ or a blue $C_{4}$.

Case 3. $|E(H)| \geq 2$.

Let $X=\left\{v_{m}, v_{m+1}, v_{m+2}\right\}$ and $Y=\left\{v_{1}, v_{2}, v_{m-2}, v_{m-1}\right\}$. All edges having one end in $X$ and one in $Y$, are colored by green or blue, otherwise we obtain a red $P_{m}$. So we obtain the complete bipartite graph $K_{3,4}$ on two partite set $X$ and $Y$ with all edges are colored green or blue. Again using Lemma 3.3, we obtain a green $P_{5}$ or a blue $C_{4}$, which completes the proof of theorem.

Corollary $3.7 R\left(P_{5}, C_{4}, P_{5}\right)=7$.

Proof. By a result in [13], $R\left(P_{5}, C_{4}, P_{4}\right)=7$ and clearly $R\left(P_{5}, C_{4}, P_{5}\right) \geq R\left(P_{5}, C_{4}, P_{4}\right)$. So it is sufficient to prove that $R\left(P_{5}, C_{4}, P_{5}\right) \leq 7$. Assume the edges of $K_{7}$ are arbitrary colored by green, blue and red. Since $R\left(P_{5}, C_{4}, P_{4}\right)=7$, we may assume that $G^{r}$ contains a copy of $P_{4}$ as a subgraph. By Lemma 3.6, $K_{7}$ must contains either a green $P_{5}$, a blue $C_{4}$ or a red $P_{5}$, which completes the proof.

Using Lemma 3.6 and Corollary 3.7, we have the following theorem.

Theorem 3.8 For all integers $m \geq 5, R\left(P_{5}, C_{4}, P_{m}\right)=m+2$.

Proof. Color all edges crossing a vertex of $K_{m}$ by green and other edges by red. Adjoin a new vertex to all vertices of colored graph $K_{m}$ and color all new edges by blue. This yields a 3-colored graph $K_{m+1}$ with no a green $P_{5}$, a blue $C_{4}$ and a red $P_{m}$ and so $R\left(P_{5}, C_{4}, P_{m}\right)>m+1$. Now assume that the edges of $K_{m+2}$ are colored with colors green, blue and red. We prove that $K_{m+2}$ contains either a green $P_{5}$, a blue $C_{4}$ or a red $P_{m}$. We prove the claim by induction on $m$. By Corollary 3.7, this claim is true when $m=5$. Assume that $R\left(P_{4}, C_{4}, P_{m-1}\right)=m+1$ for $m \geq 6$. By the induction assumption, we obtain that $K_{m+2}$ contains a red $P_{m-1}$. Using Lemma 3.6, we obtain that $K_{m+2}$ contains a green $P_{5}$, a blue $C_{4}$ or a red $P_{m}$, which completes the proof. 
Corollary 3.9 For all integers $m \geq 5, R\left(P_{4}, C_{4}, P_{m}\right)=m+2$.

Proof. Using Theorem 3.8, we have $R\left(P_{4}, C_{4}, P_{m}\right) \leq m+2$. On the other hand, the 3 -colored graph $K_{m+1}$ in the proof of Theorem 3.8, implies that $R\left(P_{4}, C_{4}, P_{m}\right)>m+1$.

Before establishing the other results of this section, we give the following lemmas which help us to calculate the Ramsey number $R\left(P_{5}, P_{5}, P_{m}\right)$.

Lemma 3.10 Let $G$ be a graph obtained by removing two edges from $K_{6}$. If each edge of $G$ is colored green or blue, then $G$ contains a monochromatic graph $P_{5}$.

Proof. By Corollary 3.2, ex $\left(6, P_{5}\right)=7$. Since $|E(G)|=13$, so without loss of generality, we may assume that $\left|E\left(G^{b}\right)\right|=6$ and $\left|E\left(G^{g}\right)\right|=7$. Since $\left|E\left(G^{b}\right)\right|=6, G^{b}$ is isomorphic to one of the graphs shown in Fig. 1. So $G^{g}$ is isomorphic to a graph obtained by removing any two edges of $\overline{G^{b}}$. One can easily check that $\overline{G^{b}}$ is isomorphic to $K_{5}-e, K_{3,3}$ or $K_{2,4}$ with one additional edge and any graph obtained by removing two edges from these graphs, still contains a $P_{5}$, which completes the proof.

Lemma 3.11 Let $G$ be a graph obtained by removing an edge from the complete bipartite graph $K_{4,5}$ with partite sets $X$ and $Y$. If each edge of $G$ is colored green or blue, then $G$ contains either a green $P_{5}$ or a blue $P_{6}$.

Proof. Let $X=\left\{x_{1}, x_{2}, x_{3}, x_{4}\right\}$ and $Y=\left\{y_{1}, y_{2}, y_{3}, y_{4}, y_{5}\right\}$. Also without loss of generality, let $e=x_{4} y_{5}$ be the edge of $K_{4,5}$ such that $G=K_{4,5}-e$. By Lemma 3.5, $G-x_{4}$ (particulary $G$ ) contains a monochromatic $P_{5}$. If $G$ contains a green $P_{5}$, we are done. So we may assume that $G$ contains a blue $P_{5}$ such as $P$. Suppose $t$ and $z$ are the end vertices of $P$. First let $t, z \in X$ and $Y \cap V(P)=\left\{y_{1}, y_{2}\right\}$. If one of the edges $t y_{i}$ or $z y_{i}$, $i \in\{3,4,5\}$, is blue we have a blue $P_{6}$. Otherwise the path $y_{3} t y_{5} z y_{4}$ is a green $P_{5}$. So let $t, z \in Y$ and $X \cap V(P)=\left\{x_{1}, x_{2}\right\}$.

Let $Y \cap V(P)=\left\{y_{1}, y_{2}, y_{3}\right\}$ such that $t=y_{1}$ and $z=y_{3}$. If one of the edges $y_{1} x_{i}$ or $y_{3} x_{i}, i \in\{3,4\}$, is blue we have a blue $P_{6}$. So we may assume that these edges are colored green. Now if one of the edges $x_{3} y_{i}, i \in\{2,4,5\}$, is green we have a green $P_{5}$. Otherwise the path $y_{5} x_{3} y_{2} x_{1} y_{3} x_{2}$ is a blue $P_{6}$. If $y_{5} \in Y \cap V(P)$, by the same argument, one can easily find either a green $P_{5}$ or a blue $P_{6}$ in $G$, which completes the proof.

In the following theorem, the values of $R\left(P_{5}, P_{5}, P_{5}\right)$ and $R\left(P_{5}, P_{5}, P_{6}\right)$ are given.

Theorem 3.12 Let $n \in\{5,6\}$. Then $R\left(P_{5}, P_{5}, P_{n}\right)=9$. 
Proof. First we prove that $R\left(P_{5}, P_{5}, P_{n}\right) \geq 9$. To see this, let $v_{1}, v_{2}, \ldots, v_{8}$ be the vertices of $K_{8}$ in the clockwise order. Let $G^{1}$ be the union of two $K_{4}$ on vertices $\left\{v_{1}, v_{2}, v_{3}, v_{4}\right\}$ and $\left\{v_{5}, v_{6}, v_{7}, v_{8}\right\}, G^{2}$ be the union of two $C_{4}$ on vertices $\left\{v_{1}, v_{5}, v_{2}, v_{6}\right\}$ and $\left\{v_{3}, v_{7}, v_{4}, v_{8}\right\}$ and $G^{3}$ be the union of two $C_{4}$ on $\left\{v_{1}, v_{7}, v_{2}, v_{8}\right\}$ and $\left\{v_{3}, v_{6}, v_{4}, v_{5}\right\}$ in this order. Color the edges of $G^{i}$ by color $i$. This gives a 3 -edge coloring of $K_{8}$ which contains no $P_{5}$ in color 1 , no $P_{5}$ in color 2 and no $P_{n}$ in color 3 . So $R\left(P_{5}, P_{5}, P_{n}\right) \geq 9$. Now we prove that $R\left(P_{5}, P_{5}, P_{n}\right) \leq 9$. Let $c: E\left(K_{9}\right) \longrightarrow\{1,2,3\}$ be an arbitrary 3-edge coloring of $K_{9}$. Also assume that $G^{i}$ denotes the spanning subgraph of $K_{9}$ induced by the edges of color $i$.

Case 1. $n=5$.

Using Corollary 3.2, we have ex $\left(9, P_{5}\right)=12$. Since $E\left(K_{9}\right)=36$, we may assume that $\left|E\left(G^{1}\right)\right|=\left|E\left(G^{2}\right)\right|=\left|E\left(G^{3}\right)\right|=12$. By Theorem 3.1, $G^{1} \cong 2 K_{4} \cup K_{1}$. This implies that $K_{4,5} \subseteq \overline{G^{1}}$. Now using Lemma 3.5 , we obtain a monochromatic $P_{5}$.

Case 2. $n=6$.

Again by Corollary $3.2, \operatorname{ex}\left(9, P_{5}\right)=12$ and $e x\left(9, P_{6}\right)=16$. If $\left|E\left(G^{1}\right)\right|=12$, by the same argument as in case 1 , we obtain that $K_{4,5} \subseteq \overline{G^{1}}$. Using Lemma 3.11, we obtain either a $P_{5}$ in color 2 or a $P_{6}$ in color 3 . Also if $\left|E\left(G^{2}\right)\right|=12$, by a similar argument, one can obtain the desired result. If $\left|E\left(G^{3}\right)\right|=16$, then Theorem 3.1 implies that $G^{3} \cong K_{5} \cup K_{4}$. Again $K_{4,5} \subseteq \overline{G^{3}}$, and hence $\overline{G^{3}}$ contains a copy of $P_{5}$ in color 1 or 2, by Lemma 3.5. Without loss of generality, we may assume that $\left|E\left(G^{1}\right)\right|=11$. Since $\left|E\left(G^{1}\right)\right|=11, G^{1}$ is not connected, otherwise we obtain a copy of $P_{5}$ in color 1 . Since $\left|E\left(G^{1}\right)\right|=11$, so there exists a component of $G^{1}$ such as $H$ such that $|H|=4$ and hence $K_{4,5} \subseteq \overline{G^{1}}$. Using Lemma 3.11, we obtain a copy of $P_{5}$ in color 2 or a copy of $P_{6}$ in color 3 , which completes the proof.

In order to determine the exact value of the Ramsey number $R\left(P_{5}, P_{5}, P_{7}\right)$, we need the following lemma which can be obtained by an argument similar to the proof of Lemma 3.6 and using Lemma 3.5 and Lemma 3.10.

Lemma 3.13 Let $m \geq 7$ and the edges of $K_{m+2}$ are colored by colors green, blue and red such that $G^{r}$ contains a copy of $P_{m-1}$ as a subgraph. Then $K_{m+2}$ contains either a green $P_{5}$, a blue $P_{5}$ or a red $P_{m}$.

As an easy consequent of Lemma 3.13, we have the following corollary.

Corollary 3.14 $R\left(P_{5}, P_{5}, P_{7}\right)=9$.

Proof. By Theorem 3.12, $R\left(P_{5}, P_{5}, P_{6}\right)=9$ and clearly $R\left(P_{5}, P_{5}, P_{7}\right) \geq R\left(P_{5}, P_{5}, P_{6}\right)$, so it is sufficient to prove that $R\left(P_{5}, P_{5}, P_{7}\right) \leq 9$. Assume that the edges of $K_{9}$ are arbitrary colored green, blue and red. Since $R\left(P_{5}, P_{5}, P_{6}\right)=9$, we may assume that $G^{r}$ contains a copy of $P_{6}$ as a subgraph. By Lemma $3.13, K_{9}$ must contains either a monochromatic $P_{5}$ in color green or blue or a red $P_{6}$, which completes the proof.

Now, we are ready to calculate the exact value of $R\left(P_{5}, P_{5}, P_{m}\right)$ for $m \geq 7$. 
Theorem 3.15 For all integers $m \geq 7, R\left(P_{5}, P_{5}, P_{m}\right)=m+2$.

Proof. Consider the graph $K_{m-1} \cup K_{2}$ and color the complete graphs $K_{m-1}$ and $K_{2}$ by color red. Consider a vertex of $K_{2}$, say $v$, and color the edges which are incident with $v$ and having another end in $K_{m-1}$ by blue and finally, color the remaining edges by green. This coloring contains neither a green $P_{5}$, a blue $P_{5}$, nor a red $P_{m}$, which means that $R\left(P_{5}, P_{5}, P_{m}\right) \geq m+2$. Now assume that the graph $K_{m+2}$ is 3 -edge colored by colors green, blue and red. We prove that $K_{m+2}$ contains either a green $P_{5}$, a blue $P_{5}$ or a red $P_{m}$. We use induction on $m$. By Corollary 3.14, the claim is true when $m=7$. Let us assume that $R\left(P_{5}, P_{5}, P_{m-1}\right) \leq m+1$ for $m \geq 8$. By the induction assumption, we obtain that $K_{m+2}$ contains a red copy of $P_{m-1}$. Using Lemma 3.13, we obtain that $K_{m+2}$ contains a green $P_{5}$, a blue $P_{5}$ or a red $P_{m}$, which completes the proof.

We need the following lemma to determine the exact value of $R\left(P_{5}, P_{6}, P_{m}\right)$.

Lemma 3.16 Let $G$ be a graph obtained by removing three edges from $K_{7}$. If each edge of $G$ is colored green or blue, then $G$ contains either a green $P_{5}$ or a blue $P_{6}$.

Proof. By Corollary 3.2, ex $\left(7, P_{5}\right)=9$ and $\operatorname{ex}\left(7, P_{6}\right)=11$. Since $|E(G)|=18$, we may assume that $\left|E\left(G^{g}\right)\right| \in\{7,8,9\}$. If $\left|E\left(G^{g}\right)\right|=9$, then by Theorem 3.1, $G^{g} \cong K_{4} \cup K_{3}$ which implies that $K_{3,4} \subseteq \overline{G^{g}}$. But removing any three edges from $K_{3,4}$, retains a copy of $P_{6}$. If $\left|E\left(G^{g}\right)\right|=7$, then $\left|E\left(G^{b}\right)\right|=11$, since $|E(G)|=18$. Now by Theorem 3.1, $G^{b} \cong K_{5} \cup K_{2}$ or $G^{b} \cong K_{2}+\bar{K}_{5}$ which implies that $K_{2,5} \subseteq \overline{G^{b}}$ or $K_{5} \subseteq \overline{G^{b}}$. But removing any three edges from $K_{2,5}$ or $K_{5}$, retains a copy of $P_{5}$. So we may assume that $\left|E\left(G^{g}\right)\right|=8$. We have the following cases.

Case 1. $G^{g}$ is connected.

Clearly $G^{g}$ contains no $C_{4}$, otherwise the connectivity of $G^{g}$ implies a copy of $P_{5}$. So $G^{g}$ contains a triangle $C$. The induced subgraph of $G^{g}$ on $V\left(K_{7}\right)-V(C)$ is an independent set, since otherwise we have a copy of $P_{5}$ in $G^{g}$. Since $\left|E\left(G^{g}\right)\right|=8$, two vertices of $C$ must contain a common neighbor outside $C$, which gives a copy of $C_{4}$ and hence a copy of $P_{5}$ in $G$.

Case 2. $G^{g}$ is disconnected.

Since ex $\left(6, P_{5}\right)=7$, ex $\left(5, P_{5}\right)=6$ by Corollary 3.2 , and $\left|E\left(G^{g}\right)\right|=8$, so $G^{g}$ can not have two components $H_{1}$ and $H_{2}$ such that $\left|V\left(H_{1}\right)\right| \leq 2$. Hence one can easily find $K_{3,4} \subseteq \overline{G^{g}}$ and clearly removing any three edges from $K_{3,4}$, retains a copy of $P_{6}$, which completes the proof.

Using Lemma 3.11 and Lemma 3.16, we have the following lemma. 
Lemma 3.17 Let $m \geq 6$ and $K_{m+3}$ is 3-edge colored with colors green, blue and red such that $G^{r}$ contains a copy of $P_{m-1}$ as a subgraph. Then $K_{m+3}$ contains either a green $P_{5}, a$ blue $P_{6}$ or a red $P_{m}$.

Proof. Assume that $v_{1}, v_{2}, \ldots, v_{m+3}$ are vertices of $K_{m+3}$ and $P=v_{1} v_{2} \ldots v_{m-1}$ is the desired copy of $P_{m-1}$ in $G^{r}$. Also let $P_{m} \not G^{r}$. We prove that $K_{m+3}$ contains either a green $P_{5}$ or a blue $P_{6}$. First assume that $v_{1} v_{m-1} \in E\left(G^{r}\right)$. If one of the vertices $v_{m}, v_{m+1}, v_{m+2}$ or $v_{m+3}$ is adjacent to $P$ by a red edge, then we obtain a red $P_{m}$. So we may assume that each edge between $\left\{v_{m}, v_{m+1}, v_{m+2}, v_{m+3}\right\}$ and $P$ is colored by green or blue. Since $m \geq 6$, we obtain a bipartite graph $K_{4,5}$ with two partite sets $X=\left\{v_{m}, v_{m+1}, v_{m+2}, v_{m+3}\right\}$ and $Y=\left\{v_{1}, v_{2}, v_{3}, v_{m-2}, v_{m-1}\right\}$ such that all edges colored green or blue and so by Lemma 3.11 , we obtain a green $P_{5}$ or a blue $P_{6}$. Hence we may assume that $v_{1} v_{m-1} \notin E\left(G^{r}\right)$. Since $P_{m} \nsubseteq G^{r}$, all edges having ends in both $\left\{v_{1}, v_{m-1}\right\}$ and $\left\{v_{m}, v_{m+1}, v_{m+2}, v_{m+3}\right\}$ are colored by green or blue. Now let $H$ be the subgraph induced by edges of color red between vertices $\left\{v_{m}, v_{m+1}, v_{m+2}, v_{m+3}\right\}$. We have the following cases.

Case 1. $|E(H)|=0$.

Since $|E(H)|=0$, then all edges among vertices $T=\left\{v_{1}, v_{m-1}, v_{m}, v_{m+1}, v_{m+2}, v_{m+3}\right\}$ are colored by green or blue. We find a vertex $v$ such that $T \cup\{v\}$ are the vertices of a complete graph on seven vertices and each edge is colored green and blue except at most three edges. If there exists a vertex $v \in P-\left\{v_{1}, v_{m-1}\right\}$ such that $v v_{i} \in E\left(G^{r}\right)$ for at most one $i \in\{m, m+1, m+2, m+3\}$, then this vertex is the desired vertex. Note that since $P_{m} \nsubseteq G^{r}$, then two consecutive vertices of $P$ are not adjacent in $G^{r}$ to a vertex in $\left\{v_{m}, v_{m+1}, v_{m+2}, v_{m+3}\right\}$. So let $v_{2} v_{i} \in E\left(G^{r}\right)$ for $i \in\{m, m+1\}$ and $v_{3} v_{i} \in E\left(G^{r}\right)$ for $i \in\{m+2, m+3\}$. Now, if $v_{3} v_{1} \in E\left(G^{r}\right)$, then $P_{m}=v_{m} v_{2} v_{1} v_{3} \ldots v_{m-1}$ is a red $P_{m}$, a contradiction. So $v_{3} v_{1} \notin E\left(G^{r}\right)$ and hence the induced subgraph on $\left\{v_{1}, v_{3}, v_{m-1}\right\}$ has at most one edge in $G^{r}$. Therefore $T \cup\left\{v_{3}\right\}$ are the vertices of a complete graph on seven vertices with at most three red edges. Using Lemma 3.16, we have either a green $P_{5}$ or a blue $P_{6}$.

Case 2. $|E(H)|=1$.

Let $v_{m} v_{m+1} \in E\left(G^{r}\right)$ be the edge of $H$ and $T=\left\{v_{1}, v_{m-1}, v_{m}, v_{m+1}, v_{m+2}, v_{m+3}\right\}$. We find a vertex $v$ such that $T \cup\{v\}$ are the vertices of a complete graph on seven vertices and each edge is colored green and blue except at most three edges. If there exists a vertex $v \in P-\left\{v_{1}, v_{m-1}\right\}$ such that $v v_{i} \notin E\left(G^{r}\right)$, for each $i \in\{m, m+1, m+2, m+3\}$, then this vertex is the desired vertex. So we assume that for some $i \in\{m, m+1, m+2, m+3\}$, $v v_{i} \in E\left(G^{r}\right)$. In $G^{r}$ the vertex $v_{2}$ (also $v_{m-2}$ ) is not adjacent to any of $v_{m}$ or $v_{m+1}$, otherwise we obtain a red $P_{m}$. So without loss of generality, let $v_{2} v_{m+2} \in E\left(G^{r}\right)$. If $v_{m-2} v_{m+2} \in E\left(G^{r}\right)$, then $v_{3} v_{i} \notin G^{r}$ for each $i \in\{m, m+1, m+2, m+3\}$, otherwise we obtain a red $P_{m}=v_{1} v_{2} v_{m+2} v_{m-2} \ldots v_{3} v_{i}$. So $v_{m+3}$ is the only vertex outside $P$ such that $v_{m-2} v_{m+3} \in E\left(G^{r}\right)$. Finally, let $v=v_{m-3}$ if $v_{1} v_{m-2} \in E\left(G^{r}\right)$ and $v=v_{m-2}$ otherwise. In 
any case, $v$ is the vertex such that $T \cup\{v\}$ are the vertices of a complete graph on seven vertices at most three red edges. Using Lemma 3.16, we obtain a green $P_{5}$ or a blue $P_{6}$.

Case 3. $|E(H)|=2$.

First let $H=2 K_{2}$, where $E(H)=\left\{v_{m} v_{m+1}, v_{m+2} v_{m+3}\right\}$. Since $P_{m} \nsubseteq G^{r}$, for each $i \in$ $\{m, m+1, m+2, m+3\}$ we have $v_{2} v_{i}, v_{m-2} v_{i} \notin E\left(G^{r}\right)$. If $v_{3} v_{i} \notin E\left(G^{r}\right)$ for each $i \in$ $\{m, m+1, m+2, m+3\}$, then we obtain the complete bipartite $K_{4,5}$ with partite set $X=\left\{v_{m}, v_{m+1}, v_{m+2}, v_{m+3}\right\}$ and $Y=\left\{v_{1}, v_{2}, v_{3}, v_{m-2}, v_{m-1}\right\}$ with all edges colored green or blue. Using Lemma 3.11, we obtain either a green $P_{5}$ or a blue $P_{6}$. So without loss of generality, we may assume that $v_{3} v_{m} \in E\left(G^{r}\right)$. Also $v_{2} v_{m-1} \notin E\left(G^{r}\right)$, otherwise we obtain a red copy of $P_{m}$. Now, $\left\{v_{1}, v_{2}, v_{m-1}, v_{m}, v_{m+1}, v_{m+2}, v_{m+3}\right\}$ are the vertices of a complete graph on seven vertices with at most three red edges. Using Lemma 3.16, we obtain either a green $P_{5}$ or a blue $P_{6}$.

Now let $H=P_{3}=v_{m} v_{m+1} v_{m+2}$. By the same argument, one can easily obtain either a complete graph on seven vertices with at most three red edges or a complete bipartite graph $K_{4,5}$ with all edges colored green or blue. Using Lemmas 3.11 and 3.16, we obtain either a green $P_{5}$ or a blue $P_{6}$.

Case 4. $|E(H)| \geq 3$.

If either $H \cong P_{4}$ or $|E(H)| \geq 4$, then all edges between $\left\{v_{1}, v_{2}, v_{3}, v_{m-2}, v_{m-1}\right\}$ and $\left\{v_{m}, v_{m+1}, v_{m+2}, v_{m+3}\right\}$ are colored by green or blue, otherwise we obtain a red copy of $P_{m}$. Since $m \geq 6$, we obtain the complete bipartite graph $K_{4,5}$ with partite set $X=$ $\left\{v_{m}, v_{m+1}, v_{m+2}, v_{m+3}\right\}$ and $Y=\left\{v_{1}, v_{2}, v_{3}, v_{m-2}, v_{m-1}\right\}$ with all edges colored green or blue. Using Lemma 3.11, we obtain either a green $P_{5}$ or a blue $P_{6}$. So it is sufficient to consider the cases that $H$ is either a star with center $v_{m}$ or the graph $K_{3} \cup K_{1}$ with isolated vertex $v_{m}$.

In the first case, all edges having end vertices in both $\left\{v_{m}, v_{m+1}, v_{m+2}, v_{m+3}\right\}$ and $\left\{v_{1}, v_{2}, v_{m-2}, v_{m-1}\right\}$ are colored green or blue, otherwise we obtain a red copy of $P_{m}$. If $v_{3} v_{i} \notin E\left(G^{r}\right), i \in\{m, m+1, m+2, m+3\}$, then we obtain the complete bipartite graph $K_{4,5}$ with partite set $X=\left\{v_{m}, v_{m+1}, v_{m+2}, v_{m+3}\right\}$ and $Y=\left\{v_{1}, v_{2}, v_{3}, v_{m-2}, v_{m-1}\right\}$ with all edges colored green or blue. Using Lemma 3.11, we obtain either a green $P_{5}$ or a blue $P_{6}$. So we may assume that $v_{3} v_{m} \in E\left(G^{r}\right)$. Now $v_{1} v_{m-2} \notin E\left(G^{r}\right)$, otherwise the path $P_{m}=v_{2} v_{1} v_{m-1} \ldots v_{3} v_{m} v_{m+1}$ is a copy of $P_{m}$ in $G^{r}$, a contradiction. Also $v_{2} v_{m-1} \notin E\left(G^{r}\right)$. Hence $\left\{v_{1}, v_{2}, v_{m-1}, v_{m-2}, v_{m+1}, v_{m+2}, v_{m+3}\right\}$ are the vertices of a complete graph on seven vertices with at most three red edges. Again using Lemma 3.16, we obtain either a green $P_{5}$ or a blue $P_{6}$.

Now let $H=K_{3} \cup K_{1}$ with isolated vertex $v_{m}$. It is clear that there is no any red edge having ends in both $\left\{v_{1}, v_{2}, v_{3}, v_{m-2}, v_{m-1}\right\}$ and $\left\{v_{m+1}, v_{m+2}, v_{m+3}\right\}$. If either $v_{2} v_{m}, v_{m-2} v_{m} \notin G^{r}$ or $v_{2} v_{m} \in G^{r}$ and $v_{m-2} v_{m} \notin G^{r}$ then $X=\left\{v_{m}, v_{m+1}, v_{m+2}, v_{m+3}\right\}$ and $Y=\left\{v_{1}, v_{2}, v_{3}, v_{m-2}, v_{m-1}\right\}$ form a complete bipartite graph $K_{4,5}$ with at most one red 
edge. Using Lemma 3.11, we obtain either a green $P_{5}$ or a blue $P_{6}$. So let both edges $v_{2} v_{m}$ and $v_{m-2} v_{m}$ be red. In this case, $v_{3} v_{1} \notin G^{r}$ otherwise $v_{m} v_{2} v_{1} v_{3} \ldots v_{m-1}$ is a copy of $P_{m}$ in $G^{r}$. Also $v_{3} v_{m-1} \notin G^{r}$, otherwise $P_{m}=v_{1} v_{2} v_{m} v_{m-2} v_{m-1} v_{3} \ldots v_{m-3}$ is a copy of $P_{m}$ in $G^{r}$. So $\left\{v_{1}, v_{3}, v_{m-1}, v_{m}, v_{m+1}, v_{m+2}, v_{m+3}\right\}$ form a $K_{7}$ with at most three red edges. Using Lemma 3.16, we obtain either a green $P_{5}$ or a blue $P_{6}$.

Corollary $3.18 R\left(P_{5}, P_{6}, P_{6}\right)=9$.

Proof. By Theorem 3.12, $R\left(P_{5}, P_{6}, P_{5}\right)=9$ and clearly $R\left(P_{5}, P_{6}, P_{6}\right) \geq R\left(P_{5}, P_{6}, P_{5}\right)$. So it is sufficient to prove $R\left(P_{5}, P_{6}, P_{6}\right) \leq 9$. Assume that the graph $K_{9}$ is 3 -edge colored by colors green, blue and red. We prove that $K_{9}$ contains either a green $P_{5}$, a blue $P_{6}$ or a red $P_{6}$. Since $R\left(P_{5}, P_{6}, P_{5}\right)=9$, so we may assume that $G^{r}$ contains a copy of $P_{5}$. Using Lemma 3.17, we obtain that $K_{9}$ contains either a green $P_{5}$, a blue $P_{6}$ or a red $P_{6}$, which completes the proof.

Finally we end this section by the following theorem.

Theorem 3.19 For all integers $m \geq 6, R\left(P_{5}, P_{6}, P_{m}\right)=m+3$.

Proof. Consider the graph $K_{m-1} \cup K_{3}$ and color the complete graphs $K_{m-1}$ and $K_{3}$ by color red. Consider two vertices of $K_{3}$, say $u, v$, and color the edges which are incident with $u$ and $v$ and having another end in $K_{m-1}$ by blue and finally, color the remaining edges by green. This coloring contains neither a green $P_{5}$, a blue $P_{6}$, nor a red $P_{m}$, so $R\left(P_{5}, P_{6}, P_{m}\right) \geq m+3$. The upper bound follows by induction on $m$. By Corollary 3.18, theorem is true when $m=6$. Let us assume that $R\left(P_{5}, P_{6}, P_{m-1}\right) \leq m+2$ for $m \geq 7$. By the induction assumption, we obtain that $K_{m+3}$ contains a red $P_{m-1}$. Using Lemma 3.17, we obtain that $K_{m+3}$ contains either a green $P_{5}$, a blue $P_{6}$ or a red $P_{m}$, which completes the proof.

Corollary 3.20 For all integers $m \geq 6, R\left(P_{4}, P_{6}, P_{m}\right)=m+3$.

\section{Multicolor Ramsey number of paths}

In this section, we give an improvement of a result of Faudree and Schelp [7] on multicolor Ramsey number $R\left(P_{n_{1}}, P_{2 n_{2}+\delta}, \ldots, P_{2 n_{t}}\right)$. In addition, we use a simple lemma to give a lower bound for the multicolor Ramsey number $R\left(P_{n_{1}}, P_{n_{2}}, \ldots, P_{n_{t}}\right)$ and we conjecture that this lower bound is the exact value of this Ramsey number if all $n_{i}$ 's are even integers greater than three. Moreover, we give some evidences for this conjecture. Before that we need a definition. By a stripe $m K_{2}$ we mean that a graph on $2 m$ vertices and $m$ independent edges. In [2], the exact value of the multicolor Ramsey number of stripes is given as follows. 
Theorem $4.1([2])$ Let $n_{1} \geq n_{2} \geq \cdots \geq n_{t}$ and $\Sigma$ denote $\sum_{i=1}^{t}\left(n_{i}-1\right)$. Then

$$
R\left(n_{1} K_{2}, n_{2} K_{2}, \ldots, n_{t} K_{2}\right)=n_{1}+\Sigma+1
$$

In the following lemma, we give a lower bound for the multicolor Ramsey number $R\left(P_{n_{1}}, P_{n_{2}}, \ldots, P_{n_{t}}\right)$.

Lemma 4.2 Assume that $G_{1}, G_{2}, \ldots, G_{t}$ are arbitrary graphs and for $i=1,2, \ldots, t$, $H_{i} \subseteq G_{i}$. Also let $n_{1} \geq n_{2} \geq \cdots \geq n_{t} \geq 3$ and $\Sigma$ denote $\Sigma_{i=1}^{t}\left(\left\lfloor\frac{n_{i}}{2}\right\rfloor-1\right)$. Then

(i) $R\left(H_{1}, H_{2}, \ldots, H_{t}\right) \leq R\left(G_{1}, G_{2}, \ldots, G_{t}\right)$,

(ii) $\left\lfloor\frac{n_{1}}{2}\right\rfloor+\Sigma+1 \leq R\left(P_{n_{1}}, P_{n_{2}}, \ldots, P_{n_{t}}\right)$,

(iii) If $n_{1}>\Sigma_{i=2}^{t}\left(\left\lfloor\frac{n_{i}}{2}\right\rfloor-1\right)$, then $n_{1}+\Sigma_{i=2}^{t}\left(\left\lfloor\frac{n_{i}}{2}\right\rfloor-1\right) \leq R\left(P_{n_{1}}, P_{n_{2}}, \ldots, P_{n_{t}}\right)$,

(iv) If $2 n_{1}>\Sigma_{i=2}^{t}\left(n_{i}-1\right)$, then $n_{1}+\Sigma_{i=1}^{t}\left(n_{i}-1\right)+1 \leq R\left(P_{2 n_{1}}, P_{2 n_{2}}, \ldots, P_{2 n_{t}}\right)$.

Proof. Part (i) is clear. Part (ii) is a direct consequent of part (i) and Theorem 4.1. To see (iii), let $m=\Sigma_{i=2}^{t}\left(\left\lfloor\frac{n_{i}}{2}\right\rfloor-1\right)$ and consider the graph $K_{n_{1}-1} \cup K_{m}$. Partition $K_{m}$ into subsets $V_{2}, V_{3}, \ldots, V_{t}$ of size $\left\lfloor\frac{n_{2}}{2}\right\rfloor-1,\left\lfloor\frac{n_{3}}{2}\right\rfloor-1, \ldots,\left\lfloor\frac{n_{t}}{2}\right\rfloor-1$, respectively. For $i=2,3, \ldots, t$, color the edges of $K_{n_{1}-1} \cup K_{m}$ having one end in $V_{i}$ and another end in $K_{n_{1}-1}$ by the $i$-th color and the remaining edges by color 1 . Clearly this coloring of $K_{n_{1}+m-1}$ contains no $P_{i}$ in color $i$, which means that part (iii) holds. Part (iv) is a direct consequent of part (iii).

The following theorem, gives an improvement of a result in [7], which follows from Theorem 2.3 and Lemma 4.2 .

Theorem 4.3 Assume that $\delta \in\{0,1\}$ and $\Sigma$ denotes $\Sigma_{i=1}^{t}\left(n_{i}-1\right)$. Then

$$
R\left(P_{2 n_{1}+\delta}, P_{2 n_{2}}, \ldots, P_{2 n_{t}}, P_{k}\right)=k+\Sigma
$$

where $k \geq \Sigma^{2}+2 \Sigma+3$ if $\delta=0$ and $k \geq 2 \Sigma^{2}+5 \Sigma+5$ otherwise.

In the following theorem, we give the exact value of some multicolor Ramsey number of paths with even number of vertices.

Theorem 4.4 Let $n_{1} \geq n_{2} \geq \cdots \geq n_{t} \geq 2$ and $m$ be positive integers. Also let $\Sigma$ denote $\sum_{i=1}^{t}\left(n_{i}-1\right)$. Then

(i) $R\left(P_{2 n_{1}}, P_{2 n_{2}}, \ldots, P_{2 n_{t}}\right)=n_{1}+\Sigma+1$ for $2 n_{1} \geq\left(\Sigma-n_{1}+2\right)^{2}+2$, 
(ii) $R\left(P_{4}, P_{4}, P_{2 m}\right)=2 m+2$ for $m \geq 2$,

(iii) $R\left(P_{4}, P_{6}, P_{2 m}\right)=2 m+3$ for $m \geq 3$,

(iv) $R\left(P_{6}, P_{6}, P_{2 m}\right)=R\left(P_{4}, P_{8}, P_{2 m}\right)=2 m+4$ for $m \geq 14$.

Proof. (i) This part is a consequent of Theorem 4.3.

(ii) First we prove that $R\left(P_{4}, P_{4}, P_{4}\right)=6$. By part (iv) of Lemma $4.2, R\left(P_{4}, P_{4}, P_{4}\right) \geq 6$. For the upper bound, let the edges of $K_{6}$ be colored by green, blue and red colors and also let $G^{g}$ be the graph induced by the green edges. Since $e x\left(6, P_{4}\right)=6$, so we may assume that $\left|E\left(G^{g}\right)\right| \leq 6$. This implies that $\overline{G^{g}}$ contains either $K_{3,3}$ or $K_{5}$ as a subgraph. If $\overline{G^{g}}$ contains a copy of $K_{5}$, we can find a copy of $P_{4}$ in blue or red, since $R\left(P_{4}, P_{4}\right)=5$. If $\overline{G^{g}}$ contains a copy of $K_{3,3}$, then it is easy to check that any two coloring of $K_{3,3}$ with colors blue and red contains a monochromatic copy of $P_{4}$. This means that $R\left(P_{4}, P_{4}, P_{4}\right) \leq 6$. For $m \geq 3$, the result follows from Corollary 3.9 and Lemma 4.2.

(iii) This part is a direct consequent of Corollary 3.20.

(iv) This part is an easy consequent of Corollary 2.5 and Lemma 4.2.

As mentioned before, it is proved that [7], $R\left(P_{n_{1}}, P_{n_{2}}, P_{n_{3}}\right)=n_{1}+\left\lfloor\frac{n_{2}}{2}\right\rfloor+\left\lfloor\frac{n_{3}}{2}\right\rfloor-2$ if $n_{1} \geq 6\left(n_{2}+n_{3}\right)^{2}$ and both $n_{2}, n_{3}$ are not odd numbers. This result can be obtained by Theorem 4.3. Theorem 4.3 shows that the lower bound in part (iii) of Lemma 4.2 is the exact value of the multicolor ramsey number $R\left(P_{n_{1}}, P_{n_{2}}, \ldots, P_{n_{t}}\right)$ if at most one of $n_{2}, n_{3}, \ldots, n_{t}$ is odd and $n_{1}$ is sufficiently large. For the case $t=4$, it seems that $R\left(P_{n_{1}}, P_{n_{2}}, P_{n_{3}}, P_{n_{4}}\right) \in\{r, r+1, r+2\}$, where $n_{1} \geq n_{2} \geq n_{3} \geq n_{4} \geq 3$ and $r=n_{1}+$ $\left\lfloor\frac{n_{2}}{2}\right\rfloor+\left\lfloor\frac{n_{3}}{2}\right\rfloor+\left\lfloor\frac{n_{4}}{2}\right\rfloor-3$. Anyway we end this paper by proposing the following conjecture, which gives the exact value of the multicolor Ramsey number of paths with even number of vertices.

Conjecture 1 For positive integers $n_{1} \geq n_{2} \geq \cdots \geq n_{t} \geq 2$, we have

$$
R\left(P_{2 n_{1}}, P_{2 n_{2}}, \ldots, P_{2 n_{t}}\right)=n_{1}+\sum_{i=1}^{t}\left(n_{i}-1\right)+1 .
$$

Theorem 4.4, gives some evidences for this conjecture. We think the following conjecture is also true, which is a generalization of the previous conjecture.

Conjecture 2 Let $n_{1} \geq n_{2} \geq \cdots \geq n_{t} \geq 4$ be positive integers such that at most one of $n_{2}, n_{3}, \ldots, n_{t}$ is odd. Then

$$
R\left(P_{n_{1}}, P_{n_{2}}, \ldots, P_{n_{t}}\right)=n_{1}+\sum_{i=2}^{t}\left(\left\lfloor\frac{n_{i}}{2}\right\rfloor-1\right) .
$$




\section{References}

[1] J. A. Bondy, U. S. R. Murty, Graph theory with applications, American Elsevier, New York, 1976.

[2] E. J. Cockayne, P. J. Lorimer, The Ramsey number for stripes, J. Austral. Math. Soc. 19 (Series A) (1975), 252-256.

[3] T. Dzido, M. Kubale, K. Piwakowski, On some Ramsey and Turán-type numbers for paths and cycles, Electron. J. Combin., \#R55 13 (2006).

[4] T. Dzido, Multicolor Ramsey numbers for paths and cycles, Discuss. Math. Graph Theory 25 (2005) 57-65.

[5] T. Dzido, R. Fidytek, On some three color Ramsey numbers for paths and cycles, Discrete Math. 309 (2009), 4955-4958

[6] P. Erdős, T. Gallai, On maximal paths and circuits of graphs, Acta Math. Acad. Sci. Hungar. 10 (1959), 33-56.

[7] R. J. Faudree, R. H. Schelp, Path Ramsey numbers in multicolorings, J. Combin. Theory, Ser. B 19 (1975), 150-160.

[8] A. Figaj, T. Luczak, The Ramsey number for a triple of long even cycles, J. Combin. Theory, Ser. B, 97 (2007), 584-596.

[9] L. Gerencsér, A. Gyárfás, On Ramsey-Type problems, Annales Universitatis Scientiarum Budapestinensis, Eötvös Sect. Math. 10 (1967), 167-170.

[10] A. Gyárfás, M. Ruszinkó, G. Sárközy, E. Szemerédi, Three-color Ramsey numbers for paths, Combinatorica 27 (1) (2007), 35-69.

[11] S. P. Radziszowski, Small Ramsey numbers, Electron. J. Combin. 1 (1994), Dynamic Surveys, DS1.12 (August 4, 2009).

[12] F. P. Ramsey, On a problem of formal logic, Proc. London Math. Soc. 2nd Ser. 30 (1930), 264-286.

[13] Z. Shao, X. Xu, X. Shi, L. Pan, Some three-color Ramsey numbers, $R\left(P_{4}, P_{5}, C_{k}\right)$ and $R\left(P_{4}, P_{6}, C_{k}\right)$, Europ. J. Combin. 30 (2009), 396-403. 